\title{
Carnets
}

Revue électronique d'études françaises de l'APEF

Deuxième série - 22 | 2021

Sous le double signe de Baudelaire et de Flaubert: traductions, adaptations, transpositions

\section{Zoltán Ambrus, l'un des premiers propagateurs de Charles Baudelaire et de Gustave Flaubert en Hongrie}

Un chapitre important des relations littéraires franco-hongroises

\section{Enikő Bauernhuber}

\section{OpenEdition}

Journals

Édition électronique

URL : https://journals.openedition.org/carnets/13217

DOI : $10.4000 /$ carnets. 13217

ISSN : 1646-7698

Éditeur

APEF

\section{Référence électronique}

Enikő Bauernhuber, «Zoltán Ambrus, l'un des premiers propagateurs de Charles Baudelaire et de Gustave Flaubert en Hongrie », Carnets [En ligne], Deuxième série - 22 | 2021, mis en ligne le 30 novembre 2021, consulté le 30 mai 2022. URL : http://journals.openedition.org/carnets/13217 ; DOI : https://doi.org/10.4000/carnets. 13217

Ce document a été généré automatiquement le 29 mai 2022.

Carnets est mis à disposition selon les termes de la licence Creative Commons - Atribution - Pas d'utilisation commerciale 4.0 International. 


\section{Zoltán Ambrus, l'un des premiers propagateurs de Charles Baudelaire et de Gustave Flaubert en Hongrie}

Un chapitre important des relations littéraires franco-hongroises

\section{Enikő Bauernhuber}

« Toutefois, il faut qu'il y ait une raison pour laquelle nos écrivains ne peuvent pas avoir une place à l'étranger. Quelle est cette raison? Nous pouvons l'apprendre premièrement par l'observation des faits suivants : quelles sont les œuvres étrangères qui ont pu devenir populaires

en dehors de leur patrie, en traduction, et, deuxièmement, en résumant ces observations, nous allons à la recherche des caractéristiques communes de ces œuvres qui ont pu obtenir des faveurs auprès du grand public. » Zoltán Ambrus, « Irodalmunk s a külföld [Notre littérature et l'étranger] » (1907) (Ambrus, 1913 :

370). ${ }^{1}$

1 Zoltán Ambrus (1861-1932), écrivain, journaliste, critique et traducteur hongrois aux talents multiples, nourrit un vif intérêt pour la littérature française pendant toute sa vie. Son œuvre, qui témoigne de riches relations avec la culture française, offre un bon exemple des liens qui se tissent entre les vies culturelles, littéraires, artistiques française et hongroise de son temps.

2 En effet, il s'agit d'une époque où les liens entre la France et la Hongrie sont très intenses, les relations littéraires et artistiques franco-hongroises sont particulièrement riches et variées. Les écrivains hongrois de la deuxième moitié du xix ${ }^{\mathrm{e}}$ siècle sont profondément attachés à la littérature française. Ils sont lecteurs et traducteurs de cette littérature dans laquelle ils puisent leurs modèles. Devançant ses contemporains, 
les écrivains-journalistes hongrois de la fin de siècle, Zoltán Ambrus est celui qui fait le plus pour faire connaître la littérature française de son temps auprès du public hongrois.

3 En tant que critique, traducteur et homme de lettres, Ambrus fut un passeur de la culture et de la littérature françaises en Hongrie permettant ainsi un transfert culturel considérable à son époque. Selon nous, Ambrus fut l'un des premiers propagateurs hongrois de Charles Baudelaire et l'un des premiers traducteurs hongrois de Gustave Flaubert. Dans notre article, nous allons développer cette idée et présenter l'activité de propagateur culturel de Zoltán Ambrus en tant que critique et traducteur hongrois de ces deux auteurs français majeurs.

\section{Préambule - motivations de recherches et actualité du sujet}

4 Nous tenons à signaler que notre présent article est rédigé à partir de notre thèse soutenue en septembre 2021 à l'Université Catholique Pázmány Péter portant sur les relations littéraires franco-hongroises et l'influence de la culture française dans les œuvres de Zoltán Ambrus (Bauernhuber, 2021). Dans cette optique, nous voudrions mettre en relief notre motivation et notre méthodologie de recherches dans ce qui suit.

Dans notre thèse, notre but est de démontrer l'influence décisive du séjour parisien (juin 1885 - janvier 1886) de Zoltán Ambrus et celle de la culture française sur son œuvre par la présentation d'éléments moins connus et moins souvent abordés, ainsi que par la mise au jour de documents de son fonds d'archives ${ }^{2}$, intéressants et révélateurs de ce point de vue. Conformément à notre objectif, le corpus de notre travail est élaboré d'après des sources moins connues ou inédites de l'œuvre de Zoltán Ambrus : d'une part, son œuvre journalistique, son œuvre de critique et de traducteur, d'autre part les documents de son fonds d'archives, actuellement en cours de traitement, de classement et d'inventorisation ${ }^{3}$, constituent le corpus de notre travail.

Suivant la méthode de recherche documentaire des fonds d'archives d'écrivains, notre travail est réalisé à partir de nos propres recherches philologiques au sein des nouvelles sources comme sa correspondance inédite, ses manuscrits, ses notes et ses brouillons autographes, ses documents officiels et personnels, ses coupures de journaux. Ce sont surtout ses notes et ses brouillons qui nous aident à déchiffrer le processus d'écriture et de traduction et à travers lesquels l'auteur nous dévoile un peu de sa création littéraire. Nous effectuons donc un travail d'historien de la littérature grâce auquel nous voulons explorer la diversité de l'œuvre et la richesse du fonds d'archives d'un écrivain hongrois tourné vers la culture française en en révélant les parties moins connues, inédites et pas encore analysées dans le but d'enrichir les recherches dans le domaine des relations littéraires franco-hongroises ${ }^{4}$.

7 L'objectif de nos recherches dans un sens plus large, c'est l'exploration de l'œuvre et du fonds d'archives de Zoltán Ambrus à travers la présentation de nouvelles sources, de données et de documents inédits, permettant de révéler ses multiples liens avec la culture française de son temps ${ }^{5}$. C'est pour cela que son rapport à Charles Baudelaire et à Gustave Flaubert que nous allons étudier dans le cadre de cet article nous semblent particulièrement importants. 


\section{L'un des premiers lecteurs hongrois de Charles Baudelaire}

8 Au début du siècle, Budapest vibre dans l'effervescence culturelle qui règne dans ses cafés $^{6}$, véritables lieux d'inspiration, vrais théâtres de la vie intellectuelle, qui servent aussi de foyer ou de refuge aux artistes et aux écrivains et qui contribuent ainsi à l'épanouissement de la littérature hongroise ${ }^{7}$. C'est une ère nouvelle qui se prépare essentiellement pendant les discussions entre écrivains autour des tables de café, comme l'un de leurs contemporains l'évoque en faisant référence à l'influence française chez Zoltán Ambrus :

Entre 1880 et 1890 , d'autres signes annonçaient aussi l'avance prochaine de la sensibilité littéraire française en Hongrie. Les jeunes écrivains en parlaient fréquemment. Un des amis de Reviczky a noté à son sujet, qu'en compagnie de Justh et de Zoltán Ambrus, lequel dans ses romans et nouvelles, devait subir l'influence de Flaubert, de Zola, et de Maupassant, ils s'entretenaient volontiers de littérature française et qu'à leur table de café, il avait été plus d'une fois question de Stendhal, de Flaubert, de Leconte de Lisle et, toujours plus souvent, de Baudelaire. (Karátson, $1969: 44)^{8}$

9 Dans la littérature critique portant sur les relations littéraires franco-hongroises ${ }^{9}$, Zoltán Ambrus est considéré comme l'un des premiers lecteurs de Baudelaire ${ }^{10}$ en Hongrie à la fin des années 1870 et l'un de ses premiers critiques en $1884^{11}$.

10 En réalité, Ambrus consacre à la littérature française l'une de ses premières critiques importantes dans laquelle il formule son avis sur les poèmes de Charles Baudelaire à propos d'un recueil d'essais de Paul Bourget dans le journal Budapesti Szemle [Revue de Budapest] en 1884, à l'âge de 23 ans. Il recourt ainsi à un procédé tout nouveau à l'époque : dans les colonnes d'un journal, il parle de la poésie de Baudelaire par le biais d'un recueil d'essais de Bourget. Il s'agit d'une caractéristique qui est essentielle dans son activitié à la fois comme journaliste et comme critique littéraire. Tout cela montre aussi à notre sens que Zoltán Ambrus dispose d'une ouverture profonde envers une nouvelle sensibilité littéraire de l'époque et qu'il réalise un transfert culturel important également par le choix de ses sujets.

11 Entre juin 1885 et janvier 1886, le jeune Ambrus séjourne à Paris et devient le correspondant parisien du journal hongrois Nemzet [Nation]. Il rédige ses chroniques, au total quinze, qui y sont publiées du 15 juin 1885 au 5 décembre 1885 sous le titre de Párizsi levél [Lettre parisienne $]^{12}$ ou Levél Párizsból [Lettre de Paris] ${ }^{13}$. Selon nos recherches récentes, la littérature française est fortement présente dans ses chroniques dans lesquelles il fait également allusion à ses lectures de Baudelaire et de Flaubert, d'ailleurs dans la même chronique ${ }^{14}$. Il est intéressant de remarquer que Zoltán Ambrus choisit le pseudonyme Flâneur pour signer ses chroniques parisiennes, ce qui fait référence évidemment à la flânerie, à la découverte de Paris en s'y baladant. Concernant ce pseudonyme, nous pouvons aussi penser à Charles Baudelaire ${ }^{15}$ et aux poèmes de son recueil Les Fleurs du Mal $(1861)^{16}$ qui faisaient partie des premières lectures d'Ambrus déjà dans les années 1870.

12 Notons que les allusions aux poèmes de Baudelaire apparaissent dans son premier recueil de nouvelles, Pókháló kisasszony [Mademoiselle Toile d'Araignée] (1893). On peut trouver également une citation du poème Semper eadem des Fleurs du Mal dans son roman d'artiste Midas király [Le Roi Midas] (publié en 1891-1892 en feuilleton, en 1906 en 
tome $)^{17}$ qui est d'ailleurs son roman le plus connu et le plus populaire auprès du public hongrois ${ }^{18}$ et qui peut être considéré comme l'expression la plus importante et la plus parfaite de l'influence française dans son œuvre littéraire selon notre conviction et d'après nos analyses. Les poèmes du recueil Les Fleurs $d u$ Mal deviennent ainsi une référence importante pour Zoltán Ambrus. Dans ses choix de critique et de traduction, il reconnaît avec la sûreté de son goût littéraire les auteurs français importants, comme Baudelaire, qu'il faut faire connaitre en Hongrie et qu'il faudra traduire en hongrois plus tard. De cette manière, il devient l'un des précurseurs de la modernité dans la littérature hongroise et ses préférences pour Baudelaire renforcent, à notre avis, ce rôle de pionnier.

\section{Le premier traducteur hongrois de Madame Bovary de Flaubert}

13 Dans ce qui suit, nous allons présenter la traduction la plus importante de Zoltán Ambrus. Il s'agit de la première traduction hongroise de Madame Bovary (1857) de Flaubert, parue d'abord en feuilleton en 1900, puis en volume en 1904. C'est dans la revue Új Magyar Szemle [Nouvel Observateur Hongrois] (1900, 1920-1921), fondée en 1900 et rédigée par Sándor Blaskovich et Zoltán Ambrus ${ }^{19}$, qu'est publiée d'abord en feuilleton la traduction de Madame Bovary par Zoltán Ambrus : d'après nos recherches, il publie entre janvier et décembre 1900, dans les douze numéros de la revue, le roman de Flaubert jusqu'au $4^{\mathrm{e}}$ chapitre de la deuxième partie en version hongroise. La traduction hongroise intégrale n'est publiée qu'en 1904 (Flaubert, 1904) en volume dans la collection Klasszikus Regénytár [Les Classiques du Roman] ${ }^{20}$. À notre sens, il s'agit de son choix de traduction le plus important grâce auquel il réalise un transfert culturel majeur à son époque et joue un rôle de passeur culturel en étant le premier à traduire en hongrois ce roman emblématique de Flaubert.

Il faut encore souligner que cette traduction hongroise ${ }^{21}$ de Madame Bovary faite par Zoltán Ambrus est publiée presque cinquante ans après sa publication en France, et que le public hongrois a pu lire ce roman de Flaubert ${ }^{22}$ dans cette traduction pendant trente années ${ }^{23}$. Cette première traduction verra le jour en quatre éditions du vivant de son traducteur $^{24}$. En 1935, c'est Sándor Hajó (1876-1944) qui traduira le roman (Flaubert, 1935) pour une collection hongroise de livre de poche. En 1943, une nouvelle traduction hongroise a été réalisée par Sándor Benamy (1899-1989) (Flaubert, 1943); et ce n'est qu'en 1958 que Albert Gyergyai (1893-1981) (Flaubert, 1958) traduira de nouveau Madame Bovary d'après la traduction de Zoltán Ambrus ${ }^{25}$. Notons pour finir et résumer ce point que la traduction la plus récente est celle de Judit Pór (1931-1995), qui date de 1993 (Flaubert, 1993).

Il faut d'ailleurs noter que les œuvres de Flaubert figuraient déjà parmi les premières lectures françaises de Zoltán Ambrus à Paris, dès 1885, ses «lettres parisiennes » en témoignent également. En effet, Flaubert incarne l'écrivain doué et doté d'un grand talent pour Ambrus, comme il en parle dans l'une de ses études qui porte sur Flaubert et qui date de 1904 (Ambrus, 1904).

16 En parcourant son recueil d'études littéraires intitulé Vezetô elmék [Les Grands esprits] (1913), reflétant son goût et ses préférences littéraires entre autres pour Honoré de Balzac, Alphonse Daudet, les Dumas, Gustave Flaubert, les Goncourt et Émile Zola ${ }^{26}$, 
nous pouvons bien voir que le maître incontesté d'Ambrus est sans nul doute Gustave Flaubert qui, selon lui, plonge au plus profond de l'âme humaine et exprime parfaitement ses pensées et ses sentiments (Ambrus, 1904: 22-23). Pour Ambrus, l'auteur de Madame Bovary (1857) était le maître des Goncourt, de Daudet, de Maupassant et de Zola. La densité de son style, les couleurs de sa langue et la musicalité de sa prose constituent d'ailleurs une tâche difficile pour son traducteur. Flaubert est un excellent critique pour les mêmes raisons : il voit toujours la beauté artistique de façon antique, avec une ardeur romantique et d'une manière toute moderne (Ambrus, $1904: 28)$.

17 Ces pensées accompagnent d'ailleurs, en tant que préface, la première traduction hongroise de Madame Bovary en 1904 dans laquelle Zoltán Ambrus développe ses idées concernant la théorie et la pratique de la traduction littéraire. Pour Ambrus, Flaubert décrit avec des mots comme le peintre peint avec un pinceau: il utilise seulement quelques mots, son style reste ainsi concis et parfait, et il trouve toujours le mot juste pour exprimer parfaitement une idée. Selon l'écrivain-traducteur, Flaubert n'a pas de mot qui est superflu: ses mots recouvrent parfaitement les notions, ses phrases suscitent des images ${ }^{27}$ (Flaubert, 1904). Sur ce point, le traducteur a une tâche difficile, car il doit redonner les images de la version originale pour que le lecteur de la traduction voie les mêmes images lors de la lecture. Dans la réflexion d'Ambrus, nous assistons à la création littéraire de Flaubert et nous pouvons voir en même temps les défis du traducteur, la mission de la traduction littéraire.

Dans la préface à sa traduction hongroise de Madame Bovary, il donne son opinion également sur la langue et le style de Flaubert, et avoue les difficultés qu'il a rencontrées lors de son activité de traducteur. Il est très important de signaler que Zoltán Ambrus, en tant que traducteur du roman de Flaubert, a réfléchi sur le texte et il a même formulé ses réflexions dans sa préface. Ce texte peut être considéré comme une sorte d'art poétique du traducteur. Ambrus y évoque un passage du texte où l'auteur parle de l'impossibilité d'exprimer les désirs, les idées, les souffrances humaines par le langage, par les mots d'une langue. D'après Ambrus, si quelqu'un est arrivé à exprimer ses sentiments et ses idées, c'était sûrement Flaubert car il est toujours précis dans ses œuvres en exprimant tout ce qu'il a imaginé, cru, senti à un niveau suprême. Comme Flaubert le dit dans l'une de ses lettres: " Tout le talent d'écrire ne consiste après tout que dans le choix des mots. C'est la précision qui fait la force. » (Flaubert, 1980 : 137).

Pour montrer la difficulté de rendre en hongrois le style flaubertien, nous citons une étude critique dans laquelle deux traducteurs hongrois de la littérature française comparent les quatre versions hongroises d'après un passage du texte original de Flaubert. Selon les traducteurs, il y a plusieurs difficultés lors de la traduction du roman de Flaubert :

Il s'agit, d'une part, de tenter de reproduire un style en hongrois qui pourrait être accepté comme 'style flaubertien', c'est-à-dire, qu'il s'agit de faire un travail minutieux, destiné à comprendre et à suivre les choix conscients de l'écrivain ; d'autre part, en entrant au cœur du texte, le traducteur devra aussi faire face à la dimension inconsciente de l'œuvre. Et, ce faisant, il finira par se rendre compte que cet immense poème en prose est en vérité un texte miné qui accumule piège sur piège. (Lőrinszky, Ádám, 2003 : 177)

Ambrus découvre aussi cette difficulté lors de la traduction. D'ailleurs, il trouve que Madame Bovary ne constitue pas une narration continue, mais plutôt une série d'images: il y a plusieurs centaines d'images sur presque 400 pages dans ce roman. 
Flaubert raconte et peint toutes les scènes de son histoire. Si quelqu'un voulait faire des illustrations pour ce roman, son imagination ne pourrait y jouer aucun rôle : il devrait juste dessiner tout ce que Flaubert a décrit avec des mots. Mais Flaubert peint seulement avec un ou deux mots, son style concis est parfait : il trouve toujours le mot ou l'expression juste pour exprimer parfaitement une idée. Dans ce roman, il y a tout un monde qui devient le miroir fidèle de toute la civilisation humaine. Selon le traducteur, un mot lui suffit parfois pour éclairer toute une scène ou pour caractériser complètement un personnage. Chez lui, les silences et la ponctuation ont aussi un sens, il y a toute une signification entre les lignes, et les dimensions de ses images sont celles d'un Shakespeare. Les couleurs de sa langue, la musicalité de sa prose donnent une tâche difficile à son traducteur. Une traduction ne pourrait donc refléter que vaguement un tel art d'écrire, précise le traducteur. La traduction la plus consciencieuse ne pourrait rendre que le sens, et ne pourrait pas faire sentir la perfection de la forme.

21 C'est pour cela que Ambrus réalise avec soin aussi cette traduction qui revêt pour lui une importance toute particulière ${ }^{28}$. Dans sa pratique de traducteur, il aspire à être fidèle à l'art d'écrire de Flaubert. Le traducteur cherche à rendre dans la version hongroise du roman de Flaubert la densité de son vocabulaire, la limpidité de son style, le rythme et la sonorité de sa prose. C'est une recherche constante de l'expression choisie qui caractérise le plus sa démarche de traducteur. Tout comme dans son œuvre d'écrivain, nous observons la rigueur d'un travail de traducteur qui tente d'approcher l'expression qui convient. Selon Ambrus, il s'agit d'une tentative qui peut réussir au niveau du sens mais qui peut rencontrer des difficultés pour ce qui est de la forme (Ambrus, 1904).

22 Après avoir évoqué la préface de la première édition hongroise du roman, nous abordons la critique contemporaine ${ }^{29}$ de Zoltán Ambrus concernant les qualités de cette version hongroise du roman de Flaubert. En 1905, Antal Radó (1862-1944) rédige la première critique (Radó, 1905) de la première traduction hongroise de Madame Bovary. D'après Radó, la traduction d'Ambrus est un chef-d'œuvre en son genre. Il loue le style, le soin, l'exactitude qui caractérisent, selon lui, le travail du traducteur. Cette critique de traduction est sans doute une étape importante dans sa réflexion sur la traduction littéraire qui arrive à son apogée dans son ouvrage théorique de 1909 (Radó, 1909).

Pour citer un détail intéressant concernant la réception de Madame Bovary en Hongrie et pour donner un exemple de transfert culturel à partir de cette traduction, nous voudrions mentionner que le prosateur hongrois Zsigmond Móricz (1879-1942) compose son roman Az Isten háta mögött [Derrière le dos de Dieu] (1911) ${ }^{30}$ (Móricz, 1939) d'après l'histoire de ce roman flaubertien en l'adaptant aux circonstances hongroises, dans un petit village hongrois. Dans le roman, nous pouvons trouver plusieurs allusions à Madame Bovary et il est possible que Móricz ait lu ce roman de Flaubert également dans la traduction de Zoltán Ambrus, même s'il parlait la langue française, et qu'il avait des projets de traduction de littérature française.

À propos de Flaubert et de la traduction de Madame Bovary, il nous semble intéressant de nous demander si le style de Flaubert a laissé son empreinte sur celui d'Ambrus. C'est sur ce point qu'il est important de citer un lecteur français de la littérature hongroise de l'époque de Zoltán Ambrus, le linguiste Aurélien Sauvageot (1897-1988), séjournant en Hongrie dans les années 1920, qui écrit ce qui suit sur le thème du rapport que les écrivains hongrois entretenaient avec leurs modèles étrangers : « Ce qui 
intéresse l'écrivain hongrois dans les littératures étrangères, ce sont les procédés d'expression, en d'autres termes : la forme, la composition, les modes de présentation d'une œuvre. » (Aurélien Sauvageot, 1961 : 13)

C'est dans son Avant-propos pour le recueil intitulé Nouvelles hongroises. Anthologie des $\mathrm{XIX}^{e}$ et $\mathrm{XX}^{e}$ siècles - publié en 1961 à Paris et contenant également une nouvelle de Zoltán Ambrus, A szerelmes gladiátor [Le gladiateur amoureux], probablement dans l'adaptation française d'Aurélien Sauvageot ${ }^{31}$ - qu'il formule cette opinion qui est la même que celle d'Ambrus. En effet, Ambrus avoue avoir subi l'influence du style de Flaubert comme il en parle dans sa correspondance et dans ses études critiques. Nous pouvons découvrir ce phénomène dans son roman le plus connu, Midas király [Le Roi Midas] : certains passages sont composés avec une exigence flaubertienne; le style impersonnel est présent dans le journal du héros, le peintre Jenő Bíró, mais en même temps, le lyrisme y est aussi (Ambrus, 1938). C'est un journal écrit d'un point de vue objectif sur la souffrance humaine, et cela représente, à notre sens, une grande valeur de ce roman d'Ambrus et montre l'influence de Flaubert dans sa création littéraire.

À ce propos, nous voudrions encore évoquer l'avis de Sauvageot, qui enseigna la langue et la littérature françaises à Budapest, au Collège Eötvös, entre 1923 et 1931. Dans les Souvenirs de ma vie hongroise (1988), Sauvageot parle une seule fois d'Ambrus, en évoquant ses années à Budapest :

Gyergyai, plus raffiné et plus sûr dans son goût, me mit en mains des œuvres de Zoltán Ambrus qui, langue mise à part, ressemblaient à s'y méprendre aux livres du même genre qui se lisaient à Paris. Je finis par comprendre que ce que s'obstinaient à vouloir mes élèves et autres conseillers, c'était me faire reconnaître que la littérature hongroise était de même niveau que la française. Or, la question n'était pas là. Ce que je voulais découvrir, c'était précisément quelque chose de différent. (Aurélien Sauvageot, 2013 : 88)

Quelques questions pertinentes se posent à partir de cette citation : à quels ouvrages de Zoltán Ambrus Aurélien Sauvageot fait-il allusion? À quelles lectures françaises de Sauvageot ces textes de Zoltán Ambrus peuvent-ils ressembler? À partir de cette constatation de Sauvageot, nous pouvons remarquer qu'il parle de l'aspiration des Hongrois qui veulent entendre que la littérature hongroise vaut autant que la littérature française, mais il lui semble beaucoup plus intéressant de révéler les différences que les similitudes entre les deux littératures. Tout de même, nous pourrions aller à la recherche d'une motivation, examinant si elle peut réellement être présente chez Ambrus. Comme nous l'avons vu jusqu'ici, c'est le style de Flaubert, mais c'est aussi celui de Maupassant, qui influencent le plus celui de Zoltán Ambrus et qui laissent sur lui leurs empreintes les plus profondes. Ambrus est d'ailleurs un maître du style limpide et de la composition claire, caractéristiques principales créant un lien de parenté entre son art d'écrire et ceux de Flaubert et de Maupassant, qui sont les écrivains qu'il apprécie le plus. Mais il s'agit chez Ambrus, selon ses propos, d'un processus inconscient, qui ne veut jamais recourir aux réminiscences littéraires. Sa conviction profonde peut se résumer ainsi : il aspire à écrire tout ce que lui suggèrent ses expériences de vie, non ses expériences de lecture. En tant qu'écrivain, Ambrus cherche à s'émanciper de toute influence littéraire, oublier ses lectures en composant ses œuvres, et s'inspirer uniquement de ses expériences, ses pensées et ses idées ${ }^{32}$. Cependant, même s'il dit tout cela dans sa correspondance, nous constatons qu'il apprend beaucoup des auteurs français cités, essentiellement au niveau de la forme, comme le confirme l'opinion d'un lecteur français, Aurélien Sauvageot. 


\section{Conclusion}

À partir de nos recherches qui nous ont fourni de nombreuses preuves concernant les liens importants de Zoltán Ambrus avec la littérature française, nous pouvons constater que sa rencontre avec la culture française influence l'ensemble de son œuvre. En parcourant son œuvre critique et son œuvre de traducteur, nous pouvons observer que la culture française y occupe la première place ${ }^{33}$. Nous pouvons aussi constater que le rôle particulier d'homme de lettres de Zoltán Ambrus dans la littérature hongroise se manifeste surtout à travers la popularisation et la traduction de grands classiques français comme Honoré de Balzac, Émile Zola $^{34}$, Gustave Flaubert ou Guy de Maupassant, mais également à travers celles d'auteurs français, comme Charles Baudelaire, qui annoncent déjà la modernité littéraire dans la littérature européenne. Ambrus est donc l'un des premiers propagateurs hongrois de Baudelaire dans ses critiques, l'un des premiers traducteurs hongrois de Flaubert et de Maupassant, le lecteur et le critique engagé de Zola pendant toute sa vie.

D'après nos analyses, les traductions de Zoltán Ambrus se caractérisent par une grande maîtrise des langues française et hongroise, par un goût et un soin parfait du style. Dans ses choix de traduction, ainsi que dans la rédaction des traductions publiées dans des séries littéraires sous sa direction, il reconnaît grace à un goût littéraire très sûr les auteurs français qu'il est important de traduire en hongrois. Selon notre conviction et d'après nos recherches, l'influence de la littérature française et celle des auteurs français importants pour Ambrus, comme Gustave Flaubert, est présente dans ses œuvres au niveau de la forme, de la composition et du style. Il s'agit d'un phénomène intéressant qui mériterait d'être analysé davantage lors de futures recherches.

À propos de son œuvre de traducteur, notamment la valeur et la qualité de ses traductions, de son goût et de son style de traducteur, nous pouvons déjà constater, comme point de départ pour les analyses à venir, que Zoltán Ambrus sert également d'intermédiaire entre les grands auteurs classiques hongrois du XIX ${ }^{\mathrm{e}}$ siècle et les futurs écrivains de la modernité dans la littérature hongroise ${ }^{35}$. En effet, Ambrus joue avant tout un rôle d'initiateur dans les lettres hongroises et participe activement aux débuts du modernisme littéraire hongrois. Ce rôle d'intermédiaire consiste donc également à initier la littérature hongroise aux nouvelles valeurs littéraires qui arrivent de la culture occidentale par l'intermédiaire du journalisme, de la critique et de la traduction en Hongrie. Il trouve donc des domaines d'activités culturelles et des moyens efficaces pour réaliser un transfert culturel important qui mène à cette initiation et à ce renouvellement de la littérature hongroise. À cet égard, les poèmes de Charles Baudelaire et surtout le roman Madame Bovary occupent une place primordiale.

31 En fin de compte, nous pouvons remarquer, à partir de notre présentation de Zoltán Ambrus comme critique de Charles Baudelaire et traducteur de Gustave Flaubert, qu'il est fortement lié à la littérature française et qu'il a beaucoup fait pour sa propagation en Hongrie par ses critiques, par ses traductions et par son important travail de rédacteur. Nous pouvons également conclure que la traduction littéraire et la rédaction des traductions est le terrain où il peut entièrement déployer une importante fonction de passeur culturel et réaliser un transfert culturel considérable. À notre avis, il joue, grâce à son orientation et son engagement pour les lettres françaises, un rôle d'initiateur et d'intermédiaire de premier ordre dans la propagation de la culture 
française en Hongrie devenant ainsi un passeur incontournable de la littérature française.

\section{BIBLIOGRAPHIE}

AMBRUS, Zoltán (1884). « A pesszimizmus egy új bírálója [Un nouveau critique du pessimisme] », Budapesti Szemle [Revue de Budapest], XxxIX, p. 143.

AMBRUS, Zoltán (1885). « Levél Párizsból [Lettre de Paris] », Nemzet [Nation], le 6 septembre 1885, pp. 5-6. Voir sur ce lien : https://adtplus.arcanum.hu/hu/view/Nemzet_1885_09/? pg =44\&layout =s\&query =Vasgy \%C3 \%A1ros (disponible le 17 octobre 2021) AMBRUS, Zoltán (1904). « Flaubert », in id.,Vezető elmék. Irodalmi karcolatok [Les Grands esprits. Esquisses littéraires]. Budapest : Révai, pp. 21-28.

AMBRUS, Zoltán (1913). « Irodalmunk s a külföld [Notre littérature et l'étranger] » (1907), in id., Vezetö elmék. Irodalmi karcolatok [Les Grands esprits. Esquisses littéraires]. Budapest : Révai, p. 370. Voir sur ce lien : https://mek.oszk.hu/08600/08654/08654.htm (disponible le 17 octobre 2021) AMBRUS, Zoltán (1916 [1906]). Midas király [Le Roi Midas], tome I, Budapest : Révai. AMBRUS, Zoltán (1938). «Le Roi Midas » (Le pressentiment - extrait), in Anthologie de la prose hongroise, par Jean Hankiss et Léopold Molnos, traductions de Pierre Barkan, Albert Gourseaud, Jean Hankiss, Edith Kubek, Jean Mezei, Léopold Molnos, Paul Rónai, Christian Sales, Paris : Éditions Sagittaire, pp. 119-122.

Ambrus Zoltán levelezése [La correspondance de Zoltán Ambrus] (1963), édition établie par Zoltán Fallenbüchl, préface par András Diószegi, Budapest : Akadémiai. Voir sur ce lien : https:// mek.oszk.hu/05900/05974/05974.htm\#15 (disponible le 25 avril 2020)

BAUDELAIRE, Charles (1996). Le Vin de Chiffonniers, in id., Les Fleurs du Mal (1861). Paris : Gallimard, coll. « folio classique ».

BAUDELAIRE, Charles (2010), Le Peintre de la vie moderne (Le Figaro, 1863). Paris : Fayard, coll. « la petite collection ».

BAUERNHUBER, Enikő (2016). « Les relations litteraires et artistiques franco-hongroises à travers les traductions de Zoltán Ambrus ", Revue de littérature comparée, n 359, 90e année, 3/2016, pp. 291306.

BAUERNHUBER, Enikő (2021). Les relations littéraires franco-hongroises et l'influence de la culture française à travers les œuvres de Zoltán Ambrus (1861-1932), thèse sous la direction de Madame Éva Martonyi, soutenue le 17 septembre 2021 à l'École Doctorale de l'Université Catholique Pázmány Péter de Hongrie. URL : https://btk.ppke.hu/uploads/articles/7430/file/ disszertacio_Bauernhuber_Eniko_2021.pdf (disponible le 17 octobre 2021) BERECZKI, Péter, Őszi, Nóra (2003). « Gustave Flaubert en Hongrie. Bibliographie », Revue d’Études françaises, Budapest : ELTE - CIEF, n 8, pp. 149-159. URL : https://www.nakala.fr//nakala/data/ $11280 /$ e2db02bb (disponible le 22 avril 2019) 
BUDA, Attila (2017). « Háborús jegyzetek a Nyugatban és más lapokban 1914-1917 [Notes de guerre dans Nyugat et dans d'autres journaux 1914-1917] ", in id., Milyen a nyár Amherstben, Budapest : Ráció, pp. 255-256. URL : http://real.mtak.hu/33328/1/BUDA_ambrus.pdf (disponible le 8 mai 2020)

BUDA, Attila (2021). « Bevezető [Introduction] », in Ambrus, Zoltán, Háborús jegyzetek 1914-1917, réd. par Attila Buda, Budapest : Napkút Kiadó, pp. 5-49.

FLAUBERT, Gustave (1857). Madame Bovary. Mœurs de province. Paris : Michel Lévy Frères. URL : https://gallica.bnf.fr/ark :/12148/btv1b8619658w/f19.image (disponible le 20 avril 2019)

FLAUBERT, Gustave (1904). Bovaryné [Madame Bovary], trad. par Zoltán Ambrus, Budapest : Révai, coll. « Classiques du Roman ».

FLAUBERT, Gustave (1935). Madame Bovary. Vidéki erkölcsök, traduit par Sándor Hajó, Budapest : Est Lapok, coll. « Filléres klasszikus regények ».

FLAUBERT, Gustave (1943). Bovaryné. Regény [Madame Bovary], trad. par Benamy Sándor, Budapest : Epocha.

FLAUBERT, Gustave (1958). Bovaryné [Madame Bovary], trad. d'Albert Gyergyai d'après la traduction de Zoltán Ambrus, Budapest : Európa.

FLAUBERT, Gustave (1993). Bovaryné [Madame Bovary], trad. par Judit Pór, Budapest: Európa.

FLAUBERT, Gustave (1980). « Lettre de Gustave Flaubert à Louise Colet, le 22 juillet 1852 », in id., Correspondance, réd. par Jean Bruneau, Paris : Gallimard, tome 2, p. 137.

GERGYE, László (2018). « Brève histoire de l'évolution du roman d'artiste hongrois depuis ses commencements jusqu'au tournant des $\mathrm{xix}^{\mathrm{e}}$ et $\mathrm{xx}^{\mathrm{e}}$ siècles ", Hungarian Studies, volume $32 / 2$, p. 278.

JózAN, Ildikó (2009). Baudelaire traduit par les poètes hongrois. Vers une théorie de la traduction, préface de Stéphane Michaud, Paris : Presses Sorbonne Nouvelle, coll. « Page ouverte ».

KARÁTSON, André (1969). Le symbolisme en Hongrie. L'influence des poétiques françaises sur la poésie hongroise dans le premier quart du $\mathrm{xx}^{e}$ siècle. Paris : PUF.

KÁLAI, Sándor (1999). « Zoltán Ambrus et la réception de Zola en Hongrie », in Lectures de Zola, réd. par Tivadar Gorilovics, Anna Szabó, Sándor Kálai, Debrecen : Debreceni Egyetem, pp. 99-115.

Ködlovagok. Irodalom és képzőművészet találkozása a századfordulón 1880-1914 [Chevaliers de la Brume La rencontre de la littérature et des beaux-arts au tournant du siècle (1880-1914)] (2012), réd. Par Gábor Palkó, Éva Kómár, Kata Kőrös, sous la direction d'Anna Cséve, choix d'images par Enikő Bauernhuber, Budapest : Petőfi Irodalmi Múzeum / Musée littéraire Petőfi.

KOROMPAY H., János (1988). Müfordítás és líraszemlélet : egy félszázad magyar Baudelaire-értelmezései [Traduction littéraire et approches de la poésie : les interprétations hongroises de Baudelaire d'un demisiècle]. Budapest : Akadémiai Kiadó.

LENGYEL, Katalin (1937). Baudelaire magyar kritikusai és fordítói [Les critiques et les traducteurs hongrois de Baudelaire]. Budapest.

Les Cafés littéraires de Budapest. Anthologie de textes littéraires hongrois et photographies anciennes (1998), préface de Gyula Zeke, textes traduits du hongrois par Joëlle Dufeuilly, Jean-Léon Muller, Chantal Philippe, Dominique Radanyi, Nantes : Le Passeur.

«Les relations littéraires entre la France et la Hongrie au xx ${ }^{\mathrm{e}}$ siècle » (2019). Colloque international organisé du 5 au 7 décembre 2018 à Budapest. Voir le numéro hors série de 2019 de 
la Revue d'Études françaises sur ce lien : http://real.mtak.hu/102095/1/Revue_2019_hors_serie.pdf (disponible le 20 janvier 2020)

LŐRINSZKY, Ildikó, Ádám, Péter (2003). « Le chapeau escamoté : étude comparée d'un passage de Madame Bovary dans quatre versions hongroises », Cahiers d'Études hongroises, Paris : Institut Hongrois, $n^{\circ} 11$, pp. 177-181.

MÁRAI, Sándor (2002). « Párizsi levél [Lettre parisienne] » (Újság, le 26 janvier 1926), in id., Tájak, városok, emberek [Paysages, villes, gens], Budapest : Helikon, pp. 152-157.

MÁRAI, Sándor (2002). « A párizsi levelező [Le correspondant parisien] » (Újság, le 22 août 1932), in id., Tájak, városok, emberek [Paysages, villes, gens], Budapest : Helikon, pp. 16-19.

MÁRAI, Sándor (2002). « Párizsi napló [Journal parisien] » (Újság, le 11 février 1933), in id., Tájak, városok, emberek [Paysages, villes, gens], Budapest : Helikon, pp. 161-165.

MARTONYI, Éva (2001). Trois siècles de relations littéraires franco-hongroises. Budapest : Akadémiai kiadó.

Mije lehetek én önnek? Ambrus Zoltán és Jászai Mari levelezése 1885-1926 [Qui suis-je pour vous ? La correspondance de Zoltán Ambrus et de Mari Jászai 1885-1926] (2021), recueillie, rédigée, annotée et introduite par Attila Buda et Rita Ackermann, traduction hongroise des passages français par Enikő Bauernhuber, Budapest : Ráció [avant parution].

MóRICZ, Zsigmond (1939). Az Isten háta mögött. Budapest : Athenaeum. Voir le texte sur ce lien : http://mek.oszk.hu/01400/01435/01435.htm (disponible le 22 avril 2019).

MóRICZ, Zsigmond (1996). Derrière le dos de Dieu. trad. par Ladislas Gara, Marcel Largeaud, Paris : Ibolya Virág.

NAGY, Péter (1980). Vous et nous. Essais de la littérature hongroise dans un contexte européen (1980). Budapest : Corvina.

Párizs nem ereszt el. Magyar írók Párizs élménye 1900-1939 [Paris ne nous a plus lâchés. L'expérience parisienne des écrivains hongrois 1900-1939] (2005). réd. par Csilla E. Csorba et Anna Cséve, Budapest : Petőfi Irodalmi Múzeum / Musée littéraire Petőfi - MTA Művészettörténeti Kutatóintézet / Académie Hongroise des Sciences.

RADó, Antal (1905). « Egy regényfordításról [D’une traduction de roman] », Magyar Nyelvőr, vol. 34, pp. 161-163. URL : http://real-j.mtak.hu/5978/1/MagyarNyelvor_1905.pdf (disponible le 20 avril 2019)

RADÓ, Antal (1909). A fordítás müvészete [L'art de la traduction]. Budapest : Franklin Társulat. URL : http://mek.oszk.hu/13600/13650/13650.pdf_(disponible le 20 avril 2019)

RőHRIG, Eszter (2009). « Flaubert látható nyelve Pór Judit Bovaryné-fordításában », Revue d’Études françaises, 15, Actes du colloque « Francia-magyar szótárak és a müfordítás (1989-2009) » organisé par le CIEF avec le soutien de l'Institut français de Budapest, les 16-18 novembre 2009 à Budapest. Textes réunis par Ildikó Lőrinszky et Dávid Szabó, pp. 43-51. URL : http://cief.elte.hu/ numero-15/numero-15/rohrig-eszter https://cief-efk.elte.hu/ref (disponible le 22 avril 2019)

SAUVAGEOT, Aurélien (1961). « Avant-propos », in Nouvelles hongroises. Anthologie des XIX ${ }^{e}$ et XX siècles, intr. par András Diószegi, préface par Aurélien Sauvageot, Paris : Éditions Seghers. SAUVAGEOT, Aurélien (2013). Souvenirs de ma vie hongroise / Magyarországi életutam. Budapest : Collège Eötvös József ELTE - Institut Français de Budapest. 
SZENTES, Éva - Hargittay, Emil (1997). Irodalmi kávéházak Pesten és Budán. Kávéház az irodalomban irodalom a kávéházban [Les cafés littéraires à Pest et à Buda. Le café dans la littérature - la littérature dans le café]. Budapest : Universitas.

TOULOUZE, Henri, Hanus, Erzsébet (2002). Bibliographie de la Hongrie en langue française, préface par Fabien Houiller, Paris - Budapest - Szeged, Institut hongrois - Bibliothèque nationale Széchényi. TÜSKÉS, Anna (2016-2020). Les relations littéraires entre la France et la Hongrie au $\mathrm{xx}^{e}$ siècle, base de données éditée à l'Institut d'Études Littéraires de l'Académie Hongroise des Sciences. https:// frhu20.iti.btk.mta.hu/levelek/ambrus-zoltan/ (disponible le 17 octobre 2021)

\section{ANNEXES}

Les traductions hongroises des œuvres françaises par Zoltán Ambrus :

BISSON, Alexandre (1898). Az államtitkár úr [Monsieur Secrétaire d'État], trad. par Zoltán Ambrus, Budapest : Vass.

BRILLAT-SAVARIN (1912). Jean-Anthelme, Az ízlés fiziológiája [La physiologie du goût], trad. par Zoltán Ambrus et Gizella Ambrus, Budapest : Singer-Wolfner.

CHERBULIEZ, Charles (1904). Victor, Feketék és vörösek [Noirs et rouges], trad. par Tivadar Lándor, Holdenis Meta, trad. et intr. par Zoltán Ambrus, Budapest : Révai, coll. «Classiques du Roman ».

CHERBULIEZ, Charles Victor (1888). Holdenis Meta, trad. par Zoltán Ambrus, Budapest : Singer-Wolfner, coll. " Collection Universelle des Romans », 2 tomes.

CHERBULIEZ, Charles Victor (1890). Miss Rovel, trad. par Zoltán Ambrus, Budapest : SingerWolfner, coll. « Collection Universelle des Romans ».

FLAUBERT, Gustave (1904). Bovaryné [Madame Bovary], trad. par Zoltán Ambrus, Budapest : Révai, coll. « Classiques du Roman ».

FRANCE, Anatole (1900). Régi dolgok [Choses anciennes], trad. par Zoltán Ambrus, Budapest : Lampel, coll. « Bibliothèque Hongroise ».

Francia elbeszélók tára. Első sorozat. [Collection des romanciers français. $1^{\text {ère }}$ série] (1898). Guy de Maupassant, Henry Gréville, Jules Richard, Eugène Chavette, Alfred Bonsergent, trad. par Zoltán Ambrus, Budapest : Lampel.

Francia elbeszélök tára. Második sorozat [Collection des romanciers français. $2^{e}$ série] (1899). Alphonse Daudet, Jules Claretie, Marcel Prévost, François Coppée, Harry Alis, Jean Malic, Ch. Epheyre, trad. par Zoltán Ambrus et Margit Tölgyessy, Budapest : Lampel.

Francia elbeszélók tára. Harmadik sorozat [Collection des romanciers français. $3^{e}$ série] (1900). Edmond About, Jules Lemaître, Villiers de l'Isle-Adam, Catulle Mendès, Maxime Paz, Alfred de Musset, Théodore Barrière et Henri Murger, trad. par Zoltán Ambrus et Sándor Hevesi, Budapest : Lampel.

Francia elbeszélők tára. Negyedik sorozat [Collection des romanciers français. $4^{e}$ série] (1901). François Coppée, René Maizeroy, Théodore de Banville, Henri Lavedan, Guy de Maupassant, trad. Géza Marquis, Andor Gábor, Béla Tóth, Budapest : Wodianer. 
Francia elbeszélók tára. Ötödik sorozat [Collection des romanciers français. $5^{e}$ série] (1905). Pierre Louys, Marcel Prévost, trad. Andor Gábor, Budapest : Franklin.

HERVIEU, Paul (1910). Ismerd meg magadat! [Peints par eux-mêmes], trad. par Zoltán Ambrus, Budapest : Lampel, coll. « Bibliothèque hongroise ».

MAUPASSANT, Guy de (1930). «A rózsakirály [Le rosier de madame Husson] », trad. par Zoltán Ambrus, in Maupassant elbeszélések [Nouvelles de Maupassant], Budapest : Franklin, pp. 73-91.

MAUPASSANT, Guy de (1930). «Az özvegy [Une veuve] », trad. par Zoltán Ambrus, in Maupassant elbeszélések [Nouvelles de Maupassant], Budapest : Franklin, pp. 66-72.

MAUPASSANT, Guy de (1930). « Gyöngy kisasszony [Mademoiselle Perle] », trad. par Zoltán Ambrus, in Maupassant elbeszélések [Nouvelles de Maupassant], Budapest : Franklin, pp. 4465.

MAUPASSANT, Guy de (1904). Gyöngy kisasszony és egyéb elbeszélések [Mademoiselle Perle et autres nouvelles], trad. par Zoltán Ambrus, Budapest : Lampel, coll. « Bibliothèque hongroise ».

SARDOU, Victorien (1898). Az agglegények [Les vieux garçons], trad. par Zoltán Ambrus, Budapest : Vass.

Les traductions hongroises des œuvres françaises du xIX ${ }^{e}$ siècle introduites par Zoltán Ambrus :

BALZAC, Honoré de (1904). Goriot apó [Père Goriot]. Grandet Eugénia [Eugénie Grandet], trad. par Frigyes Korányi fils et Sándor Hevesi, intr. par Zoltán Ambrus, Budapest : Révai, coll. «Classiques du Roman ».

BALZAC, Honoré de (1930). Grandet Eugénia [Eugénie Grandet], trad. par Géza Béry, intr. par Zoltán Ambrus, Budapest : Franklin, coll. «Classiques de l'Étranger ».

DAUDET, Alphonse (1904). Numa Roumestan. Tartarin. Tarasconi Tartarin uram jeles kalandjai [Aventures prodigieuses de Tartarin de Tarascon], trad. par Béla J. Fáy, intr. par Zoltán Ambrus, Budapest : Révai, coll. «Classiques du Roman ».

DUMAS, Alexandre fils (1907). A kaméliás hölgy [La dame aux camélias], trad. par Hugó Csergő, biographie de l'auteur par Zoltán Ambrus, Budapest : Révai, coll. « Classiques du Roman ».

FRANCE, Anatole (1930). Fehér kövön [Sur la pierre blanche], trad. par Ernő Czóbel, intr. par Zoltán Ambrus, Budapest : Révai.

GONCOURT, Edmond et Jules (1905). Demailly Károly [Charles Demailly], trad. par Frigyes Korányi fils, intr. par Zoltán Ambrus, Budapest : Révai, coll. « Classiques du Roman ». zoLA, Émile (1931). L'ÆEuvre, trad. par Győző Gergely et Andor Németh, illustrations par Jenő Zádor, introd. par Zoltán Ambrus, Budapest : Gutenberg, 2 tomes.

Les œuvres de Zoltán Ambrus traduites en français dans l'ordre chronologique de la parution :

« Le pêcheur et le marin » (1908), traduit par János Lajos Fóti, Revue de Hongrie, $1^{\text {ère }}$ année, tome I, $\mathrm{n}^{\circ} 1$, mars 1908, pp. 1-15. Réimprimé dans les Mille nouvelles nouvelles publiées par Jérôme Tharaud, Paris, 1910, ndeg. 19. 
«Septembre » (1908-1909), traduit par Maxime Beaufort, Revue de Hongrie, $1^{\text {ère }}$ année, tome II, $n^{\circ} 8$, octobre 1908, pp. 249-270, et $n^{\circ} 9$, novembre 1908, pp. 377-405, et $n^{\circ} 10$, décembre 1908 , pp. 505-529, et $2^{\mathrm{e}}$ année, tome III, $\mathrm{n}^{\circ} 1$, janvier 1909 , pp. 1-33, et $\mathrm{n}^{\circ} 2$, février 1909, pp. 137-168.

Soleil d'automne (1910), traduit par Maxime Beaufort avec une notice sur l'auteur, Bibliothèque Hongroise de la Revue de Hongrie II, Paris : Honoré Champion.

«La vraie patience de Grisélidis » (1913), traduit par Georges Delaquys et par János Lajos Fóti, Revue de Hongrie, $6^{\mathrm{e}}$ année, tome XI, $\mathrm{n}^{\circ} 1$, pp. 1-16, et $\mathrm{n}^{\circ} 2$, pp. 81-100. Réédité dans L'Echo du Danube, Budapest, juillet 1923.

« La vraie patience de Grisélidis » (1928), traduit par par Joseph Louis Fóti et Georges Delaquys, in Les maîtres conteurs hongrois, Budapest : éd. Librairie française, pp. 119-171.

« Mourants » (1936), traduit par François Gachot et Paul Rónai, Nouvelle Revue de Hongrie, $29^{\mathrm{e}}$ année, tome LIV, $\mathrm{n}^{\circ} 1$, pp. 68-75.

« Le Roi Midas » (Le pressentiment - extrait) (1938), in Anthologie de la prose hongroise, par Jean Hankiss et Léopold Molnos, traductions de Pierre Barkan, Albert Gourseaud, Jean Hankiss, Edith Kubek, Jean Mezei, Léopold Molnos, Paul Rónai, Christian Sales, Paris : Éditions Sagittaire, pp. 119-122.

«L'enfant prodigue » (1943), sans le nom du traducteur, Nouvelle Revue de Hongrie, $36^{\mathrm{e}}$ année, tome LXIX, $\mathrm{n}^{\circ}$ 7, pp. 91-96.

« Le gladiateur amoureux » (1961), adapté par Aurélien Sauvageot, sans le nom du traducteur, in Nouvelles hongroises. Anthologie des XIX et XX ${ }^{e}$ siècles, intr. par András Diószegi, préface par Aurélien Sauvageot, Paris : Éditions Seghers, pp. 77-83.

\section{NOTES}

1. Étant donné que la majeure partie de l'œuvre et de la correspondance de Zoltán Ambrus n'est pas traduite en français, là où le nom du traducteur n'est pas indiqué, nous donnons notre propre traduction dans le texte. Pour ce qui est des traductions de ses œuvres en français, voir notre bibliographie établie d'après nos recherches dans l'annexe. Voir encore à ce propos Toulouze et Hanus (2002).

2. Le fonds d'archives de Zoltán Ambrus est gardé dans le Département des Manuscrits de la Bibliothèque nationale Széchényi sous la cote Fonds 471.

3. Voir à ce propos Buda $(2017,2021)$.

4. Voir à ce propos Nagy (1980); Martonyi (2001); Csorba et Cséve (2005); Revue d'Études françaises (numéro hors série 2019) ; Tüskés (2016-2020).

5. Voir à ce propos notre précédent article : Bauernhuber (2016).

6. Voir Szentes - Hargittay (1997).

7. Voir l'anthologie intitulée Les Cafés littéraires de Budapest (1998).

8. Ce souvenir de Pál Koroda est rapporté par Katalin Lengyel (Lengyel 1937 : 12).

9. Voir Korompay (1988: 52). Voir également la thèse d'Ildikó Józan (2009).

10. Notons que la première critique hongroise sur la poésie de Baudelaire est rédigée par Sándor Hegedűs dans Fóvárosi Lapok [Journaux de la Capitale] en 1869 et que la première traduction hongroise de Baudelaire est faite par Sándor Endrődi en 1889 (Lengyel, 1937). 
11. Voir Ambrus (1884). Dans cet article, il parle aussi de Baudelaire à propos du recueil Essais de psychologie contemporaine (Baudelaire, Renan, Flaubert, Taine, Stendhal) de Paul Bourget.

12. Notons que l'écrivain hongrois Sándor Márai (1900-1989), qui apprécie beaucoup d'ailleurs la prose de Zoltán Ambrus, rédige également des articles de ses séjours parisiens sous le même titre (Márai, 2002).

13. Les chroniques parisiennes de Zoltán Ambrus seront publiées dans le recueil suivant: Mije lehetek én önnek? Ambrus Zoltán és Jászai Mari levelezése 1885-1926 [Qui suis-je pour vous? La correspondance de Zoltán Ambrus et de Mari Jászai 1885-1926] (2021, avant parution).

14. Il s'agit de la huitième chronique (Ambrus, 1885).

15. Rappelons l'idée suivante de Charles Baudelaire : « Pour le parfait flâneur, pour l'observateur passionné, c'est une immense jouissance que d'élire domicile dans le nombre, dans l'ondoyant, dans le mouvement, dans le fugitif et l'infini. Être hors de chez soi, et pourtant se sentir partout chez soi ; voir le monde, être au centre du monde et rester caché au monde » (Baudelaire, 2010 : 112).

16. Notamment son poème intitulé Le Vin de Chiffonniers, in Baudelaire (1996 : 142).

17. Le passage du poème Semper eadem de Baudelaire cité en français dans le texte hongrois l'illustre bien : «D'où vous vient, disiez-vous, cette tristesse étrange. Montant comme la mer sur le roc noir et nu? / Quand notre cœur a fait une fois sa vendange, Vivre est un mal ! (Ambrus, 1916 : 94). Le texte intégral du roman de Zoltán Ambrus est disponible sur ce lien: https:// mek.oszk.hu/05200/05286/05286.htm (disponible le 20 avril 2019).

18. Voir à ce propos Gergye (2018).

19. Pour la version en ligne de la revue, voir https://adtplus.arcanum.hu/hu/collection/ UjMagyarSzemle/ (disponible le 20 avril 2019)

20. Voir la lettre de Géza Voinovich à Zoltán Ambrus, du 7 juillet 1904 (Ambrus, 1963 : lettre num. 152).

21. Voir la lettre de la maison d'édition des Frères Révai, adressée à Ambrus, le novembre 1904, concernant les honoraires de la traduction et la préface d'Ambrus de Madame Bovary (Ambrus, 1963 : lettre num. 153).

22. Pour la réception de Flaubert en Hongrie, voir Bereczki - Őszi (2003).

23. Voir deux articles parmi les coupures de journaux dans le Fonds de Zoltán Ambrus, Fonds 471 à la Bibliothèque nationale Széchényi de Budapest : "Flaubert als Romantiker ", Sonntagsbeilage num. 7. zur [... ?] Zeitung, Berlin, le 18 février 1917, num. 89. et « La servante de Madame Bovary », sans références.

24. Deuxième édition en 1914, troisième édition en 1920, quatrième édition en 1924.

25. Voir aussi la lettre d'Albert Gyergyai concernant sa traduction de L'Éducation sentimentale de Flaubert, adressée à Zoltán Ambrus, du 15 septembre 1925, in Ambrus, 1963 : lettre num. 433. et la réponse de Zoltán Ambrus, lettre num. 434.

26. Pour les références, voir la $2^{\mathrm{e}}$ partie de notre bibliographie sélective dans l'annexe: «Les traductions hongroises des œuvres françaises du XIXe siècle introduites par Zoltán Ambrus ».

27. Voir le texte intégral de la première traduction hongroise du roman et la préface de Zoltán Ambrus sur ce lien : http://mek.oszk.hu/09500/09550/09550.htm (disponible le 20 avril 2019).

28. Selon les notes biographiques de sa fille, Gizella Ambrus, gardées dans le Fonds Zoltán Ambrus à la Bibliothèque nationale Széchényi (Fonds 471), cette traduction est très importante pour l'écrivain. Nous donnons notre propre traduction française de ces notes dans ce qui suit: « 1900-ban, az Új Magyar Szemle számára kezdi meg, 3 év múlva újra előveszi, rengeteg időt és fáradtságot áldozva rá. Hosszan eltűnődik egy-egy kifejezésen, egy-egy apró részleten ; a regény jeleneteit, a leírt helyeket és tárgyakat igyekszik elképzelni. Annyira magával ragadja ez a munka, hogy alig képes mással foglalkozni. Bár már régóta jól ismeri a regényt, az most is rendkívül nagy hatást tesz rá. Mint maga elbeszélte : mikor az Emma haláláról szóló részt fordította - éjjel a kávéházban - a könnyei folytak a meghatottságtól. [Il la commence en 1900 
pour la revue Nouvel Observateur Hongrois. 3 années après, il la recommence et y consacre énormément de temps et d'énergie. Il réfléchit pendant longtemps sur une expression, un détail ; il cherche à imaginer les scènes du roman, les lieux et les objets décrits. Il est tellement saisi par ce travail qu'il n'est guère capable de s'occuper d'autres choses. Bien qu'il connaisse depuis longtemps le roman, celui-ci exerce sur lui une influence extraordinaire. Comme il l'a raconté : lorsqu'il a traduit le passage portant sur la mort d'Emma - pendant la nuit au café - ses larmes coulaient d'émotion.]».

29. Voir le compte rendu de la revue Vasárnapi Újság [Journal de Dimanche], 1907, 54e année, num. 24, p. 488.

30. Voir la préface de Béla M. Pogány dans la revue Nyugat [Occident] en 1927 pour la traduction française du roman de Móricz faite par Ladislas Gara et Marcel Largeaud, publié en $1927 \mathrm{chez}$ Rieder à Paris : http://epa.oszk.hu/00000/00022/00420/13119.htm (disponible le 22 avril 2019).

31. Voir la lettre adressée à Zoltán Fallenbüchl le 3 septembre 1960 concernant la traduction française de cette nouvelle de Zoltán Ambrus dans un recueil français, édité sous l'égide de l'UNESCO, dans le Fonds de Zoltán Ambrus à la Bibliothèque nationale Széchényi : Fonds 471.

32. Voir sa lettre, datant du 25 mai 1914, adressée à Frigyes Riedl, in Ambrus (1963 : 231).

33. Voir notre bibliographie sélective dans l'annexe.

34. Voir à ce propos Kálai (1999).

35. Voir, entre autres, à ce propos le recueil d'études Ködlovagok. Irodalom és képzőművészet találkozása a századfordulón 1880-1914 [Chevaliers de la Brume - La rencontre de la littérature et des beaux-arts au tournant du siècle (1880-1914)] (2012).

\section{RÉSUMÉS}

Dans notre article, la présence de Charles Baudelaire et celle de Gustave Flaubert en Hongrie sont examinées et présentées à travers l'activité de propagateur culturel de Zoltán Ambrus (18611932), écrivain, journaliste, critique et traducteur hongrois aux talents multiples qui nourrit un vif intérêt pour la littérature française. Son œuvre, qui témoigne de riches relations avec la culture française, offre un bon exemple des liens qui se tissent entre les vies culturelles française et hongroise de la fin du XIX ${ }^{\mathrm{e}}$ siècle. Ambrus est également un traducteur important et un critique éminent de son époque. Il est parmi les premiers critiques hongrois qui font référence au recueil Les Fleurs du Mal (1861) de Baudelaire dans les années 1880. Sa traduction la plus importante est celle de Madame Bovary (1857) de Flaubert, la première traduction hongroise du roman flaubertien, publiée en 1904 en Hongrie. Son attachement profond à la littérature française se manifeste dans ses œuvres littéraires, ses critiques et ses traductions.

The presence of Charles Baudelaire and Gustave Flaubert in Hungary is studied through the oeuvre of a Hungarian writer, Zoltán Ambrus (1861-1932) in our paper. Zoltán Ambrus is a versatile Hungarian author keenly interested in French literature, whose journalistic, reviewing, translating oeuvre is rich in French aspects: he provides a good example on how the French and Hungarian artistic and literary lives are interwoven in several ways. Ambrus, an outstanding literary critic and translator of his age, was amoung the first critics to refer to Baudelaire's Les Fleurs du Mal (1861) in the 1880s and the first to translate Flaubert's Madame Bovary (1857) into Hungarian in 1904. His French taste and education, profound knowledge of French literature are manifested in his oeuvre, translations and prose works. 
INDEX

Keywords : Ambrus (Zoltán), Hungarian-French literary relations, translation, critics, cultural transfer

Mots-clés : Ambrus (Zoltán), relations littéraires franco-hongroises, traduction, critiques, transfert culturel

\section{AUTEUR}

\section{ENIKŐ BAUERNHUBER}

Université Catholique Pázmány Péter, Hongrie

eniko.bauernhuber[at]gmail.com 